\title{
Release of the angiogenesis inhibitor angiostatin in patients with proliferative diabetic retinopathy: association with retinal photocoagulation
}

\author{
J.Spranger ${ }^{1}$, H.-P.Hammes ${ }^{2}$, K. T. Preissner ${ }^{3}$, H.Schatz ${ }^{4}$, A.F.H.Pfeiffer ${ }^{1}$ \\ ${ }^{1}$ Department of Clinical Nutrition, German Institute of Human Nutrition Potsdam-Rehbrücke, Bergholz-Rehbrücke, Germany \\ ${ }^{2}$ Department of Internal Medicine, University Hospital Mannheim, Mannheim Germany \\ ${ }^{3}$ Department of Biochemistry, Justus-Liebig-University, Gießen, Germany \\ ${ }^{4}$ Department of Internal Medicine, University Hospital Bergmannsheil, University of Bochum, Bochum, Germany
}

\section{Abstract}

Aims/hypothesis. Proliferative diabetic retinopathy is a major debilitating disease causing most cases of blindness in humans in the Western world. Photocoagulation is the established therapy of proliferative diabetic retinopathy, although the molecular mechanisms of its effects are still not known. Recently angiostatin has been characterized as a potent inhibitor of neovascularization. Apart from a possible downregulation of angiogenic cytokines, release of angiostatin could initiate the anti-angiogenic effects of retinal photocoagulation.

Methods. We investigated the regulation of angiostatin and the angiogenic cytokines vascular endothelial growth factor and basic fibroblast growth factor in vivo by comparing vitreal concentrations of 18 control patients and 34 patients with proliferative diabetic retinopathy with and without previous photocoagulation. Concentrations of basic fibroblast growth factor and angiostatin were additionally measured in serum, while vascular endothelial growth factor is known to be regulated locally in the eye. Cytokines were measured by immunological methods.

Results. Angiostatin could be detected in 2 out of 18 control patients and in 25 out of 34 diabetic patients $(p<0.00001)$. Most importantly, production of angiostatin in human vitreous correlated significantly with previous retinal photocoagulation $(p<0.0001)$ in patients with proliferative diabetic retinopathy. Only two patients (one control and one diabetic) had detectable serum concentrations of angiostatin. Additionally patients with proliferative diabetic retinopathy and with previous photocoagulation had significantly lower concentrations of vascular endothelial growth factor $(0.9 \pm 0.1 \mathrm{ng} / \mathrm{ml} ; p<0.0001)$ than diabetic patients without previous photocoagulation $(4.0 \pm 0.8 \mathrm{ng} / \mathrm{ml})$. The investigation of vitreal and serum basic fibroblast growth factor concentrations yielded no significant differences between the groups.

Conclusion/interpretation. Angiostatin is not a regularly expressed angiogenesis inhibitor in human vitreous. The alterations we observed suggest that local release of angiostatin and down-regulation of vascular endothelial growth factor mediate the therapeutic effects of retinal photocoagulation in proliferative diabetic retinopathy. [Diabetologia (2000) 43:1404-1407]

Keywords Diabetes, angiostatin, VEGF, bFGF, angiogenesis, retinopathy, cytokine, growth factors, photocoagulation, retina.
Received: 23 June 2000 and in revised form: 27 July 2000

Corresponding author: A.F.H.Pfeiffer, MD, German Institute of Human Nutrition Potsdam-Rehbrücke, Department of Clinical Nutrition, Arthur-Scheunert-Allee 114-116, 14558 Bergholz-Rehbrücke, Germany

Abbreviations: bFGF, Basic fibroblast growth factor; PDR, proliferative diabetic retinopathy; NVD, new vessels on the disc; NVE, new vessels elsewhere; VEGF, vascular endothelial growth factor.
Proliferative diabetic retinopathy (PDR) and macular degeneration are the most common causes of blindness in industrialised nations. Retinal neovascularization is the main feature of PDR and the angiogenic activity produced by the retina seems to be diffusable because extensive capillary non-perfusion is associated with rubeosis iridis, thus producing angiogenesis as far anterior as the surface of the iris. Accordingly increased vitreal concentrations of angio- 
genic growth factors such as vascular endothelial growth factor (VEGF) and IGF-I $[1,2]$ have been shown to correlate with the degree of retinal ischaemia in PDR.

Retinal scatter photocoagulation often prevents further retinal neovascularization and has been shown to be associated with a reduction in the incidence of severe visual loss and retinal neovascularization [3]. The molecular mechanisms of the angiogenesis inhibitory effects of retinal photocoagulation are, however, not known. The switch to an angiogenic phenotype of tumours requires both up-regulation of angiogenic stimulators and down-regulation of angiogenesis inhibitors. Vice versa anti-angiogenic effects of retinal photocoagulation might be mediated by an increased expression of an angiogenesis inhibitor or down-regulation of angiogenic growth factors such as VEGF or basic fibroblast growth factor (bFGF). A reduction of VEGF in the vitreous after retinal photocoagulation was shown in six patients indicating that a reduced expression of angiogenic growth factors might be responsible for the positive therapeutic effects [1]. The number of patients with subsequent measurement of VEGF was, however, small in this study and vitreous was re-examined for VEGF after dilution of the vitreous in the first vitrectomy [1]. Therefore dilution might explain the reduction of VEGF in the patients who were investigated.

Recently angiostatin, a fragment encompassing the kringle region of plasminogen, has been identified and characterized as a potent inhibitor of neovascularization [4]. Differential production of angiostatin and the angiogenic growth factors VEGF and bFGF is a potential mechanism of the positive therapeutic effects of retinal photocoagulation. Angiostatin, VEGF and bFGF were measured in the vitreous of patients without an underlying proliferative retinal disease and in patients with proliferative diabetic retinopathy with and without previous scatter photocoagulation surgery to investigate changes in the release of these proteins in vivo. Only angiostatin and bFGF were measured in parall in the serum of patients, because it is known that intraocular changes of VEGF are caused primarily by local production [5]. Therefore VEGF was not measured in the circulation.

\section{Subjects and methods}

The study was approved by the ethics committee of the University of Bochum and informed consent was obtained from the patients at least one day before surgery. Between the groups patients were similar in age and sex ratio. Groups of patients with diabetes mellitus did not show statistically significant differences regarding duration of diabetes, occurrence of hypertension or metabolic control measured by $\mathrm{HbA}_{1 \mathrm{c}}$ (Table 1). Patients with retinal proliferations were excluded from the control group. Of the patients with PDR and previous pho-
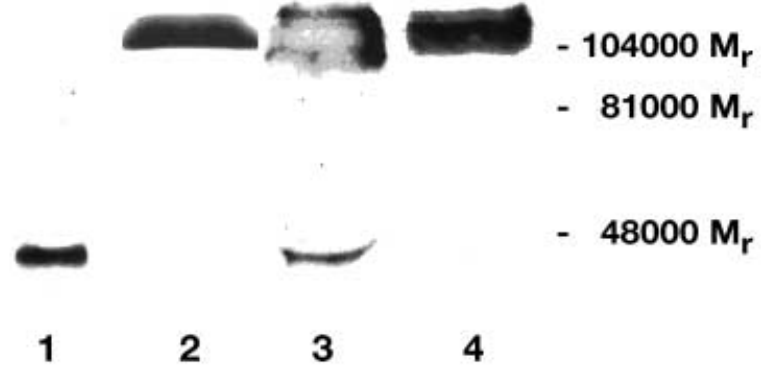

Fig. 1. Western blots with a polyclonal plasminogen antibody. Control lanes with angiostatin of $43000 \mathrm{M}_{\mathrm{r}}$ produced by elastase digestion of human plasminogen (Lane 1) and with human plasminogen (Lane 2). Apart from the strong plasminogen signal, a protein of $43000 \mathrm{M}_{\mathrm{r}}$, corresponding to the predicted molecular mass of angiostatin could be detected in human vitreous (Lane 3). Partial quenching of the plasminogen signal is most likely due to the large amounts of plasminogen and did not occur after competition with angiostatin. The band of $43000 \mathrm{M}_{\mathrm{r}}$ did not appear after preincubation of the antibody with angiostatin K1-4 fragment thereby showing specificity of reaction. The plasminogen signal is not competed with by angiostatin because epitopes present on plasminogen but not on angiostatin are recognized by the polyclonal antibody (Lane 4)

tocoagulation eight had new vessels elsewhere (NVE), eight had new vessels on the disk (NVD) and ten patients had NVE and NVD. Of the patients with PDR but without previous photocoagulation two had NVE, two had NVD and four had NVE and NVD.

Vitreous was obtained during pars plana vitrectomy. Undiluted samples of vitreous fluid were aspirated at the beginning of surgery and transferred immediately to a plastic tube. The samples were cooled on ice and centrifuged at $14000 \mathrm{~g}$ for $15 \mathrm{~min}$ at $4^{\circ} \mathrm{C}$. Aliquots were then stored at $-80^{\circ} \mathrm{C}$ until assay. Haemorrhagic samples were excluded from the analysis. Peripheral blood samples were withdrawn before surgery from resting patients and allowed to clot for $30 \mathrm{~min}$ before centrifugation at $1000 \mathrm{~g}$ for $10 \mathrm{~min}$ at $4{ }^{\circ} \mathrm{C}$. Serum was then removed and stored at $-80^{\circ} \mathrm{C}$ until assay.

Angiostatin was detected by western blotting using plasminogen-specific, polyclonal antibodies (Dako, Hamburg, Germany). A protein of $43000 \mathrm{M}_{\mathrm{r}}$, corresponding to the predicted molecular mass of angiostatin kringle fragment 1-4 could be detected. The K1-4 band did not appear after pre-incubation of the antibody with a previously isolated angiostatin K1-4 fragment thereby showing specificity of reaction (Fig. 1). Briefly, the angiostatin fragment was prepared by elastase digestion of human plasminogen. The proteolytically cleaved fragments were purified by affinity chromatography on lysine-Sepharose columns. Fragments were examined by SDS-polyacrylamide gel electrophoresis. Blots were analysed with automatic band identification by laser densitometry using Image QuaNT software (Molecular Dynamics, Sunnyvale, Calif., USA) with predefined sensitivity, noise value and Kernel relation, thereby obtaining observer-independent measurements of the blots. Total protein was measured by coomassie brilliant blue assay. All samples had similar amounts of total protein thereby showing that negative results were not due to low protein content. VEGF and bFGF were measured by enzyme-linked immuno sorbent assays (R\&D, Minneapolis, Minn., USA). Assays were done according to the manufacturer's instructions. The intra-assay and inter-assay coefficients of variation were 4.1 and $6.7 \%$ for the VEGF assay and $6.8 \%$ and $7.3 \%$ for the 


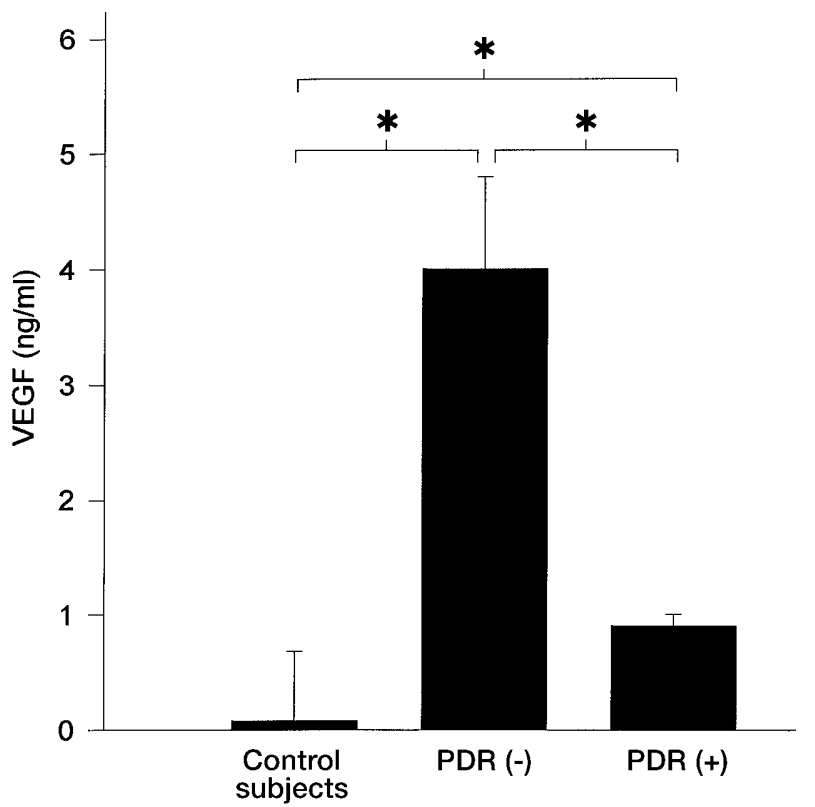

Fig. 2. Concentrations of Vascular Endothelial Growth Factor (VEGF) in controls and patients with proliferative diabetic retinopathy with [PDR $(+)]$ and without previous retinal photocoagulation $[\mathrm{PDR}(-)] . * p<0.0001$

bFGF assay, respectively. Additionally samples containing various concentrations of an external bFGF-standard of the National Institute for Biological Standards and Control (NIBSC) were measured in the bFGF assay showing an excellent correlation with the internal standards of the assay.

Data are reported as means \pm SEM. The symmetry of the data was calculated by the method of David, Pearson and Stephens [6]. The Mann-Whitney $U$ test and the chi-square test were used for comparisons of groups. A $p$ value of less than 0.05 was considered to be significant.

\section{Results}

The anti-angiogenic protein angiostatin was detectable in vitreous of 25 of 34 (74\%) patients with PDR but only in 2 of $18(11 \%)$ control patients $(p<0.0001)$. Most importantly 24 of $26(92 \%)$ patients with PDR and with previous scatter photocoagulation had detectable angiostatin concentrations in vitreous compared with 3 of $26(12 \%)$ patients without previous photocoagulation including control patients $(p<0.0001)$. Only 1 of $8(12.5 \%)$ patients with PDR but without retinal photocoagulation had de- tectable angiostatin concentrations in vitreous, which was similar to the ratio in control patients suggesting that angiostatin production is functionally associated with previous retinal scatter photocoagulation rather than with PDR (Table 1). Only two patients (one control patient and one patient with PDR) had detectable angiostatin concentrations in serum. The angiostatin precursor plasminogen was detectable in considerable amount in all samples investigated.

Vitreous concentrations of human VEGF were increased in patients with proliferative diabetic retinopathy $(1.5 \pm 0.2 \mathrm{ng} / \mathrm{ml} ; p=0.0001)$ compared with control patients $(0.08 \pm 0.6 \mathrm{ng} / \mathrm{ml})$. Similar changes have been shown previously [1]. Most notable patients with PDR and with previous photocoagulation had lower concentrations of VEGF $(0.9 \pm 0.1 \mathrm{ng} / \mathrm{ml}$; $p<0.0001)$ than diabetic patients without previous photocoagulation $(4.0 \pm 0.8 \mathrm{ng} / \mathrm{ml})$. Concentrations of VEGF were, however, still tenfold increased in patients with PDR and with previous photocoagulation compared with control patients $(p<0.0001)$ (Fig. 2).

The investigation of bFGF yielded no significant differences of the mean concentrations in vitreous and serum of patients with PDR (vitreous: $0.7 \pm 0.1$ $\mathrm{pg} / \mathrm{ml}$; serum: $5.1 \pm 1.3 \mathrm{pg} / \mathrm{ml}$ ) with previous photocoagulation (vitreous: $0.7 \pm 0.2 \mathrm{pg} / \mathrm{ml}$; serum: $5.0 \pm 1.5$ $\mathrm{pg} / \mathrm{ml}$ ), without previous photocoagulation (vitreous: $0.9 \pm 0.4 \mathrm{pg} / \mathrm{ml}$; serum: $5.9 \pm 3.2 \mathrm{pg} / \mathrm{ml}$ ) and control patients (vitreous: $0.7 \pm 0.2 \mathrm{pg} / \mathrm{ml}$; serum: $3.5 \pm 1.1$ $\mathrm{pg} / \mathrm{ml})$.

\section{Discussion}

In the mammalian eye, vessels are normally excluded from the vitreous, a compartment which has been shown to have anti-angiogenic activity. Only 2 out of 18 control patients had detectable concentrations of angiostatin. Therefore angiostatin does not appear to be a regularly expressed angiogenesis inhibitor in the eye.

Our study shows an association between release of the angiogenesis inhibitor angiostatin and decreased production of the angiogenic growth factor VEGF in the eye with previous retinal scatter photocoagulation. Regulation of these changes seems to occur locally, because angiostatin was not measurable in relevant amounts in human serum, the angiostatin pre-

Table 1. Patients characteristics and occurrence of angiostatin in control patients and patients with proliferative diabetic retinopathy

\begin{tabular}{|c|c|c|c|c|c|c|c|c|}
\hline \multirow[t]{2}{*}{ Subjects } & \multirow[t]{2}{*}{$n$} & \multirow{2}{*}{$\begin{array}{l}\text { Age } \\
\text { (year) }\end{array}$} & \multirow[t]{2}{*}{ Women } & \multirow[t]{2}{*}{ Men } & \multirow{2}{*}{$\begin{array}{l}\text { Duration of } \\
\text { diabetes (year) }\end{array}$} & \multirow{2}{*}{$\begin{array}{l}\mathrm{HbA}_{1 \mathrm{c}} \\
(\%)\end{array}$} & \multicolumn{2}{|c|}{ Angiostatin } \\
\hline & & & & & & & negative & positive \\
\hline Control subjects & 18 & $68.7 \pm 3.7$ & 10 & 8 & - & $5.1 \pm 0.1$ & 16 & 2 \\
\hline - with photocoagulation & 26 & $63.2 \pm 6.8$ & 13 & 13 & $18.8 \pm 2$ & $8.1 \pm 0.2$ & 2 & 24 \\
\hline - without photocoagulation & 8 & $60.6 \pm 2.5$ & 4 & 4 & $15.5 \pm 4.5$ & $7.7 \pm 0.7$ & 7 & 1 \\
\hline
\end{tabular}


cursor plasminogen could be found in any of the samples investigated and VEGF is known to be produced locally. These data suggest that release of angiostatin and a decreased production of VEGF are possible mediators of the anti-angiogenic effects of retinal scatter photocoagulation in proliferative diabetic retinopathy. The patients in our study still, however, had intraocular proliferative activity requiring pars plana vitrectomy. Angiostatin might not be sufficient to neutralize the effect of the tenfold increased concentrations of VEGF, which could explain that photocoagulation was unable to prevent surgery in the patients we investigated. Additionally other growth factors could considerably influence the reappearance of intraocular proliferations after retinal photocoagulation. The regulation of angiostatin release is not clear. This study provides data that plasminogen occurs in considerable amounts in the vitreous of control and diabetes patients. Preliminary data of our group showed relevant amounts of the angiostatin precursor plasminogen in retinae of healthy wistar rats (unpublished data). Recently a macrophage-derived metalloproteinase has been shown to generate angiostatin in Lewis Lung carcinoma [7]. Possibly a similar process with activation of macrophages, expression of macrophage-derived metalloelastase and production of angiostatin from pre-existing plasminogen occurs in the retina after retinal photocoagulation. Whether angiostatin is generated only after scatter photocoagulation or whether a similar mechanism occurs after focal photocoagulation and might explain at least in part its positive therapeutic effects is not clear.

Our study found a strong association between intraocular release of the anti-angiogenic substance angiostatin, decreased expression of the angiogenic growth factor VEGF and previous retinal photocoagulation in patients with proliferative diabetic retinopathy. Clearly the release of an angiogenesis inhibitor offers a mechanism to explain the therapeutic effects of retinal photocoagulation, which is the established treatment of choice in patients with PDR. It is not yet possible, however, to directly establish whether angiostatin and VEGF inhibition are clinically useful and can eventually reduce visual loss in patients with proliferative diabetic retinopathy without the known adverse effects of extensive retinal scatter photocoagulation on visual acuity and visual field.

Acknowledgements. J. Spranger was supported by the German Diabetes Association.

\section{References}

1. Aiello LP, Avery RL, Arigg PG et al. (1994) Vascular endothelial growth factor in ocular fluid of patients with diabetic retinopathy and other retinal disorders. N Engl J Med 331: 1480-1487

2. Meyer-Schwickerath R, Pfeiffer A, Blum WF et al. (1993) Vitreous levels of the insulin-like growth factors I and II, and the insulin-like growth factor binding proteins 2 and 3, increase in neovascular eye disease. Studies in nondiabetic and diabetic subjects. J Clin Invest 92: 2620-2625

3. Early Treatment Diabetic Retinopathy Study Research Group (1991) Early Photocoagulation for Diabetic Retinopathy: ETDRS Report Number 9. Ophthalmology 98 [Suppl]: 766-785

4. O'Reilly MS, Holmgren L, Shing Y et al. (1994) Angiostatin: a novel angiogenesis inhibitor that mediates the suppression of metastases by a lewis lung carcinoma. Cell 79(2): 315-328

5. Burgos R, Simo R, Audi L et al. (1997) Vitreous levels of vascular endothelial growth factor are not influenced by its serum concentrations in diabetic retinopathy. Diabetologia 40(9): 1107-1109

6. Ramm B, Hofmann G (1987) Biomathematik. 3rd edn. Ferdinand Enke Verlag, Stuttgart

7. Dong Z, Kumar R, Yang X, Fidler IJ (1997) Macrophagederived metalloelastase is responsible for the generation of angiostatin in lewis lung carcinoma. Cell 88: 801-810 